\title{
EchoGéo
}

38 | 2016

Varia

\section{L'Alaska Railroad Corporation : un modèle ferroviaire original aux confins des États-Unis entre diversification commerciale et stratégie touristique intégrée}

\section{Matthieu Schorung}

\section{OpenEdition}

\section{Journals}

Electronic version

URL: https://journals.openedition.org/echogeo/14807

DOI: 10.4000/echogeo.14807

ISSN: 1963-1197

Publisher

Pôle de recherche pour l'organisation et la diffusion de l'information géographique (CNRS UMR 8586)

Electronic reference

Matthieu Schorung, "L'Alaska Railroad Corporation : un modèle ferroviaire original aux confins des États-Unis entre diversification commerciale et stratégie touristique intégrée ", EchoGéo [Online], 38 | 2016, Online since 13 December 2016, connection on 31 July 2021. URL: http:// journals.openedition.org/echogeo/14807 ; DOI: https://doi.org/10.4000/echogeo.14807

This text was automatically generated on 31 July 2021

EchoGéo est mis à disposition selon les termes de la licence Creative Commons Attribution - Pas d'Utilisation Commerciale - Pas de Modification 4.0 International (CC BY-NC-ND) 


\title{
L'Alaska Railroad Corporation : un modèle ferroviaire original aux confins des États-Unis entre diversification commerciale et stratégie touristique intégrée
}

\author{
Matthieu Schorung
}

1 Le transport ferroviaire de passagers connaît depuis le milieu du $\mathrm{XX}^{\mathrm{e}}$ siècle une crise majeure aux États-Unis engendrée par la concurrence de modes de transports qui répondent aux nouvelles demandes de la société étatsunienne. Malgré la création d'Amtrak en 1970 et la fréquentation en hausse de ses lignes - notamment régionales depuis près de deux décennies, le rail connaît des difficultés structurelles renforcées par un manque de soutien politique et financier (Perl, 2002; Wolmar, 2012 ; USDOT, 2016). Ce dynamisme ferroviaire se concentre dans quelques régions - Nord-Est, Californie, Nord-Ouest - et repose surtout sur les services régionaux d'Amtrak. À ceci s'ajoute une complexité institutionnelle et opérationnelle propre aux États-Unis qui retarde un retour du train dans les déplacements interurbains. En effet, le réseau de chemins de fer étatsunien étant détenu par les entreprises de fret et celles-ci assurant également la gestion de l'activité ferroviaire, le fonctionnement du secteur dépend de ces entreprises privées.

2 L'Alaska constitue un cas à part aux États-Unis. Cet État ne dispose que d'une ligne ferroviaire desservant le territoire selon un axe méridien. Le gouvernement fédéral a possédé et exploité l'Alaska Railroad Company de 1914 à 1985. La ligne est d'abord destinée à ouvrir le territoire alaskien au développement économique et à accélérer l'exploitation de minerais. À la suite du vote de l'Alaska Railroad Transfer Act en 1982, l'État fédéral transfère la responsabilité de l'Alaska Railroad Corporation (ARRC) à l'État de l'Alaska, et cette dernière devient donc une compagnie publique relevant de l'État ${ }^{1}$. L'ARRC, seule compagnie à fournir des services ferroviaires de fret et de passagers en 
Alaska, en dessert les villes principales - Whittier, Seward, Anchorage et Fairbanks. Sur l'ensemble du territoire américain, les activités de fret et de passagers sont séparées et le transport interurbain de voyageurs relève d'Amtrak. L'Alaska offre le seul exemple de compagnie publique ayant la responsabilité intégrale d'une ligne - infrastructure, matériel roulant, organisation du service, investissements, gestion opérationnelle - et reposant sur l'unicité des services, ainsi que celui d'une compagnie de fret qui continue à exploiter des trains pour les voyageurs. Au-delà de ces caractéristiques d'organisation et de fonctionnement spécifiques, il est intéressant de relever les actions mises en œuvre par l'ARRC pour développer ses services de voyageurs notamment une stratégie touristique avancée. La compagnie devient alors un acteur à part entière de l'activité touristique en Alaska.

3 Le développement des chemins de fer et l'histoire de l'Alaska Railroad Corporation ont été surtout analysés dans le cadre d'ouvrages historiques ou de rapports institutionnels (Fitch, 1967 ; Clifford, 1999 ; Gale Cengage Learning, 2004 ; Tuck et Killorin, 2004 ; Schultz, 2012; Osborne, 2013) et représentés dans des histoires illustrées visant davantage à valoriser les paysages somptueux de l'Alaska (Cohen, 1984 ; Clifford, 1999). Des travaux d'histoire générale de l'Alaska traitent du rôle des transports et en particulier des chemins de fer dans l'ouverture et le développement de ce territoire (Sherwood, 1967 ; Madden, 2005). Dans le champ de recherche consacré aux stratégies touristiques propres à un acteur de transport, quelques travaux sont consacrés au cas étatsunien (Gordon, 1996; Shaffer, 2001; Gay, 2006; Stilgoe, 2007; Wolmar, 2012). Toutefois, la littérature comporte des lacunes quant à l'analyse de l'ARRC aujourd'hui, notamment à l'aune de sa stratégie de développement comprenant un volet touristique essentiel.

Cet article propose de s'intéresser à un territoire souvent peu pris en compte dans la littérature en langue française et d'étudier les caractéristiques propres à l'Alaska Railroad Corporation. La ligne ferroviaire est aujourd'hui le support d'une stratégie touristique intégrée visant à diversifier les revenus de la compagnie. Alors que l'implantation de la grande vitesse ferroviaire est particulièrement compliquée aux États-Unis et que ce renouveau ferroviaire est confronté à de lourds obstacles institutionnels, politiques et financiers, avoir un regard décentré sur l'expérience alaskienne pourrait apporter des pistes de réflexion pour l'ensemble du pays. Il s'agit d'une étude de cas régional destinée à appréhender les spécificités de l'organisation ferroviaire de l'Alaska qui s'inscrit dans une démarche de recherche plus globale sur le fonctionnement du réseau ferroviaire des États-Unis. Le cas alaskien qui repose sur une compagnie ferroviaire de dimension régionale, dite de classe $\mathrm{II}^{2}$, étonne par ses caractéristiques de fonctionnement et sa stratégie commerciale. Cet article repose sur une analyse approfondie de la littérature grise - rapports financiers et fiches synthétiques de l'ARRC, documents institutionnels de l'État concernant la compagnie ainsi que du site internet de la compagnie et des brochures touristiques proposées par la compagnie. En quoi le mode d'organisation du modèle ferroviaire contribue au dynamisme ferroviaire alaskien? Dans quelle mesure cette dynamique ferroviaire actuelle s'appuie-t-elle sur l'émergence de l'ARRC comme véritable acteur du tourisme?

5 Dans un premier temps, nous reviendrons sur la structuration historique du territoire de l'Alaska par la construction du réseau, pour ensuite nous intéresser à l'élaboration d'un modèle ferroviaire unique aux États-Unis. Puis dans un troisième temps, nous 
analyserons la nouvelle dynamique ferroviaire de l'Alaska fondée sur une stratégie touristique avancée.

\section{Une mise en valeur et une structuration du territoire par la construction du réseau selon des logiques économiques}

6 L'Alaska Railroad Corporation est d'abord le fruit d'un engagement d'acteurs privés au début du XX $\mathrm{X}^{\mathrm{e}}$ siècle. En 1903, la construction de la première ligne d'Alaska est achevée par des investisseurs privés de Seattle, sous le nom d'Alaska Central Railway, afin de relier les petites villes minières de Klondike, Fairbanks et Nome ${ }^{3}$. L'Alaska attire nombre d'intérêts privés depuis la découverte de filons d'or et de charbon au centre et au nord. Cet engouement minier permet d'ouvrir le territoire alaskien au peuplement et au développement depuis les ports libres de glace du golfe d'Alaska. Cependant, la crise financière de 1907 et les coûts très élevés de construction et de maintenance poussent l'Alaska Central Railway à la faillite. L'entreprise est réorganisée en 1910 et ne gère plus qu'un réseau de 82 kilomètres. Une fois encore, l'ampleur de la tâche, le marché potentiel très limité et les spécificités du relief et du climat la conduisent à nouveau à la faillite (ADOT\&PF, 2015).

7 C'est à ce moment-là que l'État fédéral décide de s'y intéresser et de reprendre en main cette entreprise. En 1912, le Congrès vote l'Alaska Territorial Act qui prévoit la mise en place d'une commission d'évaluation du réseau de transports de l'Alaska. Lors de la remise de son rapport, elle conclut qu'un chemin de fer transalaskien est nécessaire pour l'économie de l'État. Le Congrès décide en 1914 d'approuver le financement et la construction d'un réseau de 1600 kilomètres pour ouvrir le territoire et faciliter l'exploitation minière. Le président $\mathrm{W}$. Wilson choisit le tracé central qui reprend la ligne existante et relie Seward à Fairbanks. En 1923, au prix d'efforts logistiques exceptionnels, le tronçon ferroviaire principal de 850 kilomètres entre ces deux villes est achevé. La construction du chemin de fer bouleverse la géographie de l'Alaska: construction de lignes télégraphiques puis téléphoniques, aménagement de terminaux portuaires pour réceptionner les matériaux, installation de centaines d'ouvriers au cœur de l'Alaska, ouverture et exploitation de mines de charbon et de fonderies, fondation de nouvelles villes pour l'installation de ces ouvriers et de leurs familles, et desserte des communautés rurales et minières du centre et du nord (Schultz, 2012).

$\mathrm{Au}$ cours du XXe siècle, le chemin de fer perd progressivement son monopole surtout pour le transport de passagers, dans l'ensemble du pays. Le train ne peut alors rivaliser avec les avantages de la voiture individuelle, du camion, puis de l'avion. Cela, couplé à une politique fédérale très volontariste en faveur du mode autoroutier, ouvre une période de crise profonde du secteur ferroviaire dès les années 1920, crise qui s'aggrave après 1945. L'Alaska Railroad Corporation rencontre les mêmes difficultés que partout ailleurs aux États-Unis en plus de la perte d'une grande partie du fret militaire liée à la fin de la guerre. En outre, une catastrophe naturelle déstabilise profondément l'entreprise. En 1964, un tremblement de terre d'une violence exceptionnelle endommage toutes les infrastructures ferroviaires et portuaires. Au début des années 1970, l'administration Nixon émet l'idée d'un transfert de gestion de l'ARRC de l'État fédéral à l'État d'Alaska pour se décharger d'une entité lourde à gérer et dont les 
performances diminuent depuis la fin de la Seconde Guerre mondiale. Ce projet est finalement mis en œuvre sous l'administration Reagan. Il est alors décidé de transférer intégralement l'ARRC à l'État de l'Alaska (ADOT\&PF, 2015).

9 Le train en Alaska, comme dans les Grandes Plaines et l'Ouest américain, a permis d'ouvrir le territoire au peuplement et à l'exploitation. La structuration du territoire a donc été soutenue par des intérêts économiques avec l'Alaska Railroad comme vecteur de transformation du territoire. Le statut de l'ARRC évolue, suivant des logiques politiques, et émerge alors un modèle ferroviaire particulier, en comparaison de la situation dans le reste des États-Unis.

\section{L'élaboration d'un modèle ferroviaire inédit aux confins des États-Unis}

\section{Le transfert à l'État de l'Alaska : premier élément d'un modèle ferroviaire unique aux États-Unis}

L'Alaska Railroad Transfer Act est voté en 1982, et le 5 janvier 1985, la responsabilité de l'entreprise est transférée à l'État de l'Alaska. L'ARRC devient une entreprise indépendante à but lucratif jouissant d'une personnalité morale, détenue par l'État de l'Alaska, chargée de gérer, d'exploiter et d'assurer la maintenance du réseau ferroviaire alaskien Elle ne reçoit aucune dotation de fonctionnement de l'État et ne bénéficie que de crédits d'investissements. Il lui est autorisé de candidater à des fonds fédéraux pour des projets d'investissements lourds. L'ARRC est dirigée par un directoire de sept membres, nommés par le gouverneur de l'État (ARRC, 2014).

Elle doit gérer en priorité les décennies de sous-investissement fédéral, surtout depuis les travaux engagés à la suite du tremblement de terre de 1965. Les services de fret assurent l'essentiel de l'activité et des revenus de l'Alaska Railroad. En 1988, 90 \% des revenus sont générés par cette branche. Toutefois, à partir des années 1990, l'ARRC cherche à diversifier ses activités pour moins dépendre du fret, particulièrement sensible aux cours du pétrole et de l'énergie. Le mode ferroviaire cherche à accompagner les mutations économiques de l'Alaska afin de préserver son rôle dans le système de transports de l'État.

Après l'obtention de crédits fédéraux d'investissements en 1996, l'ARRC s'engage dans un programme pluriannuel de modernisation de son réseau et de son matériel roulant nouvelles locomotives, nouvelles rames pour les voyageurs, remplacement de ballasts, modernisation de certains terminaux et dépôts, informatisation des systèmes de répartition, nouvel arrêt au Denali National Park. Les investissements sont poursuivis tout au long des années 2000 pour maintenir l'attractivité du mode ferroviaire et pour répondre à une croissance continue du trafic de marchandises et de voyageurs. Des projets existent pour construire une liaison ferroviaire terrestre continue entre l'Alaska et le reste des États-Unis en passant par les provinces canadiennes du Yukon et de Colombie-Britannique. Mais le coût du projet et le refus d'engagement de gouvernement canadien, notamment des gouvernements Chrétien (1993-2004) et Martin (2004-2006), conduisent pour le moment à une impasse ${ }^{4}$.

13 Alors que la responsabilité des lignes régionales d'Amtrak est progressivement transférée aux États depuis la loi fédérale PRIIA votée en 2008, l'Alaska offre un 
exemple abouti de transfert complet d'un service de transports à l'État fédéré. Ce statut constitue la première caractéristique majeure de ce modèle ferroviaire, dont l'élaboration repose également sur la pleine prise en compte des problématiques ferroviaires dans les politiques de l'État.

\section{Une compagnie indépendante mais pleinement intégrée dans la planification de l'État}

14 L'État de l'Alaska reconnaît un quadruple rôle à l'ARRC : un rôle économique dans le système global d'échanges de l'État, un rôle touristique pour les croisiéristes, un rôle environnemental de lutte contre la congestion routière, et enfin un rôle social par la desserte de communautés rurales non desservies par la route (ADOT\&PF, 2015). C'est pour cela que dans ses documents de planification, l'État présente des objectifs plus généraux associés à d'autres secteurs de l'action publique: développement économique, politique énergétique, essor des réseaux de transports urbains. L'échelon fédéré est le chef de file de la politique ferroviaire en Alaska bien que la compagnie soit statutairement indépendante (ADOT\&PF, 2008). L'ARRC fait partie intégrante de la réflexion et de la planification sur la politique de transports de l'État (illustration 1).

Illustration 1 - Les objectifs de l'État de l'Alaska pour l'ARRC : une intégration aux autres politiques étatiques

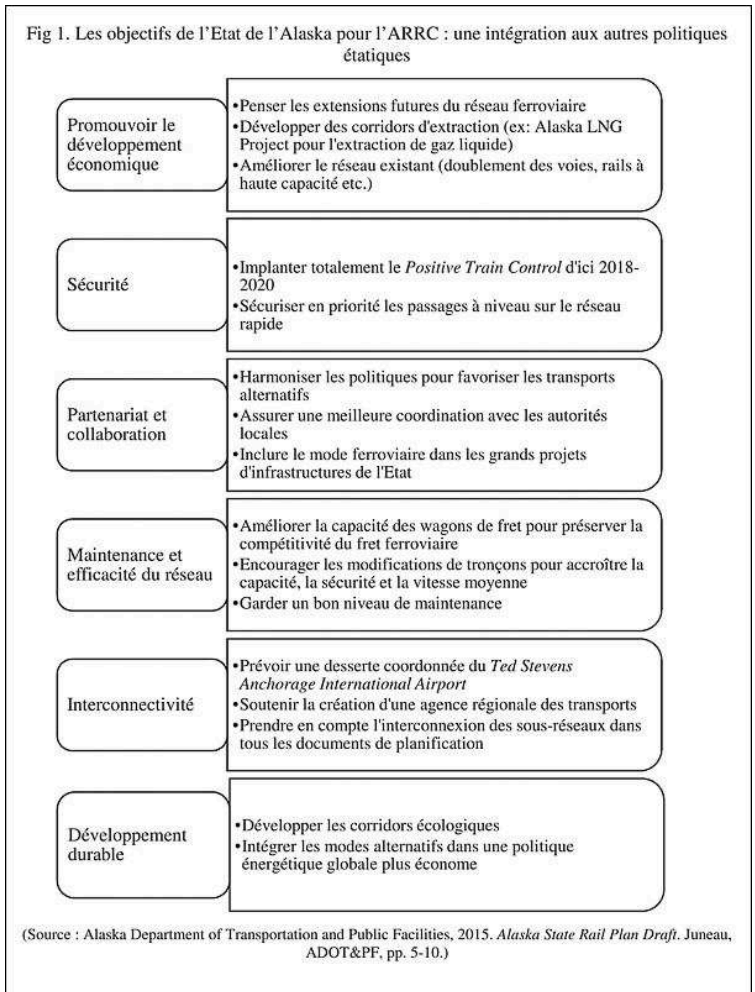

Source : Alaska Department of Transportation and Public Facilities, 2015. Alaska State Rail Plan Draft. Juneau, ADOT\&PF, p. 5-10.)

Dans les documents institutionnels étatiques, le mode ferroviaire est pleinement mis en rapport avec les autres modes de transports. Du fait de la configuration géophysique du territoire alaskien, les grandes infrastructures de transports terrestres sont concentrées en un nombre limité de corridors. Le réseau autoroutier et routier est 
plutôt développé dans la partie centrale et méridionale de l'État, alors que d'autres en sont dépourvues. Certains tronçons autoroutiers présentent des premiers signes de congestion aux heures de pointe, d'après le département des Transports. L'Alaska dépend également de son système aéroportuaire, notamment pour accueillir le tourisme international. Les deux principaux aéroports - Ted Stevens Anchorage International et Fairbanks International - sont en train de mettre à jour leur plan directeur, et il est précisé qu'ils ne connaîtront pas de problème de capacité d'ici vingt ans (ADOT\&PF, 2015). Le mode aéroportuaire ne constitue pas un mode concurrent pour l'Alaska Railroad, puisque le trafic de ce dernier se concentre sur des trajets courts ou moyens. À l'inverse, les autorités mettent en avant la complémentarité future entre cette ligne ferroviaire interurbaine et les réseaux de transports urbains qui pourraient être développés. L'ARRC soutient d'ailleurs la création d'une autorité régionale des transports (ARRC, 2002).

Illustration 2- Le réseau de l'Alaska Railroad et le système de transports de l'Alaska

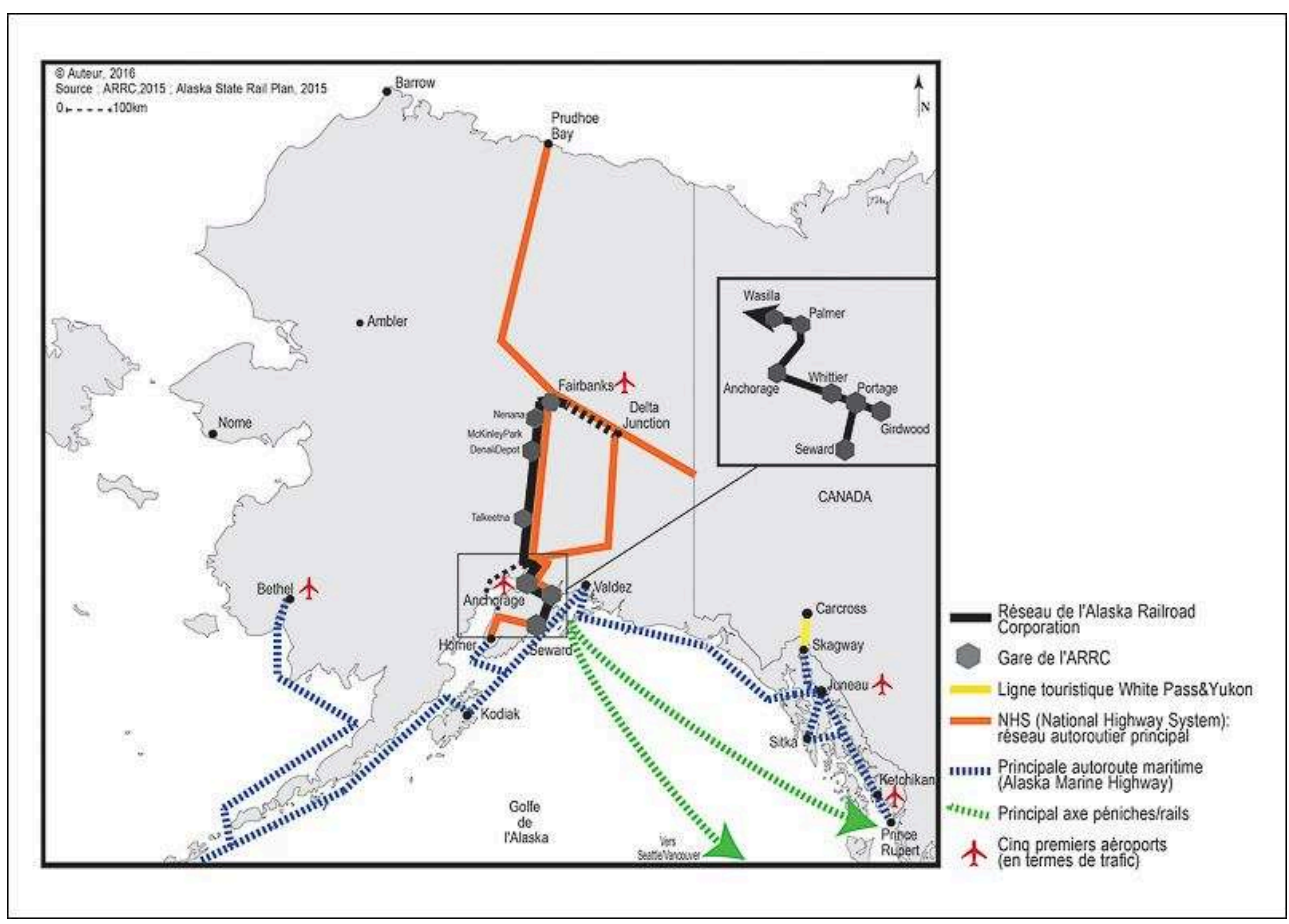

Le réseau de l'Alaska Railroad apparaît comme un axe central du système de transports alaskien, assurant la liaison entre le nord et le sud de l'État, et entre son cœur économique et urbain et le reste du continent nord-américain (illustration 2). Son intégration avec les autres modes - autoroutes, terminaux de ferries, voies maritimes, aéroports - est de plus en plus cruciale pour assurer son développement, son attractivité et sa bonne santé financière. Le transfert de l'entière responsabilité de l'ARRC à l'échelon fédéré semble avoir été bénéfique pour l'entreprise, et l'État l'a progressivement intégrée dans ses réflexions sur l'avenir du système de transports et sur l'essor économique de l'Alaska plus généralement. Mais ces éléments ne permettent pas d'expliquer directement le dynamisme de cette ligne ferroviaire: le mode d'organisation du secteur ferroviaire et la stratégie commerciale de la compagnie sont à prendre en compte. 


\section{Unité des services, diversification des revenus et stratégie touristique : les clés du succès?}

\section{Une gestion intégrée des services de fret et de passagers}

17 Aujourd'hui, s'applique aux États-Unis une intégration verticale complète du réseau ferroviaire. Les compagnies privées de fret organisent et décident comme elles l'entendent les activités ferroviaires, l'attribution des sillons, les opérations de maintenance ainsi que les investissements (Thompson et Kohon, 2012; Gallamore et Meyer, 2014).

L'Alaska Railroad Corporation est une compagnie de fret ferroviaire de classe II qui possède et exploite la quasi-intégralité du réseau ferroviaire de l'Alaska soit 1055 kilomètres $(\mathrm{ADOT \& PF}, 2015)^{5}$. Au-delà de son statut, l'ARRC est unique en son genre aux États-Unis puisque les services sont unifiés et l'intégration verticale complète. Elle exploite et gère à la fois le transport de marchandises et celui de passagers, et possède l'infrastructure, les équipements ferroviaires et le matériel roulant (ARRC, 2013) ${ }^{6}$. Cette unicité des services lui assure une gestion optimisée des sillons et du trafic. Il n'existe pas de difficultés majeures, selon la compagnie ou le département des transports de l'État de l'Alaska, pour faire cohabiter des trains de marchandises et de voyageurs. Cette situation s'explique, au-delà de l'organisation des services, par le nombre restreint de sillons à attribuer aux trains de voyageurs par jour et par saison ainsi que par la configuration du réseau composé essentiellement d'un tronçon principal. C'est d'abord la fréquentation modeste, bien que non négligeable en termes de revenus et d'image commerciale, de l'ARRC par les voyageurs qui explique que la cohabitation des différents services ne pose pas de difficultés majeures.

19 Comme pour le reste des compagnies de classes II et III aux États-Unis, le réseau est orienté vers le fret. Le réseau de fret étatsunien est considéré comme l'un des plus dynamiques du monde. En 2013, les entreprises ferroviaires de classe I ont généré 67,6 milliards de dollars de revenus, 4 milliards de dollars pour celles de classes II et III. $91 \%$ du transport par rail concerne les marchandises en vrac et les matières premières - produits agricoles, matériaux de construction, charbon, minerais, papier, produits chimiques - alors que les $9 \%$ restants relèvent du trafic intermodal et concernent essentiellement des produits transformés. Le chemin de fer est utilisé pour des moyennes et des longues distances, en particulier pour le transport de marchandises de côte à côte (Association of American Railroads, 2014). L'activité de l'ARRC repose d'abord sur le transport de marchandises puisque $61 \%$ des revenus d'exploitation en 2015 proviennent de cette branche (ARRC, 2016).

L'ARRC vante son rôle central dans le système de transports de l'État et les acteurs politiques reconnaissent également le rôle structurant de cet axe ferroviaire (Brehmer, 2015). Par rapport à sa population - 710000 habitants en $2010^{7}$ - la fréquentation de l'Alaska Railroad est plus qu'honorable, d'autant que le réseau routier y est relativement limité, que des petites communautés n'ont pas accès à la route et que seules $20 \%$ des routes d'Alaska sont goudronnées, contre plus de $90 \%$ en moyenne dans les autres États. Les services assurés en hiver offrent une desserte ponctuelle quand les niveaux d'enneigement rendent les routes impraticables. Toutefois, on note dans les documents de l'ARRC une absence de réflexion sur la composition de sa fréquentation: part des touristes et des résidents, répartition entre trajets courts et complets, etc. 
21 Elle est considérée aujourd'hui comme une compagnie en bonne santé financière avec des revenus nets relativement stables : 13,4 millions de dollars de revenus nets en 2010, 12,5 millions en 2012 et 14,1 en 2014 (ARRC, 2014). On constate en outre la bonne tenue de la fréquentation de la ligne - 468700 passagers en 2014 - bien que ces résultats n'atteignent pas le pic historique de l'année 2007 - 564800 passagers. À l'inverse, le secteur du fret connait une érosion continue de son activité en tonnage bien qu'il assure toujours près de la moitié des revenus de la compagnie (ARRC, 2015a). Cela est dû au ralentissement de l'industrie charbonnière et à une moindre consommation de pétrole conventionnel au profit des sables bitumineux. C'est d'ailleurs pour cela que la compagnie cherche à faire évoluer ses activités de fret vers des produits à plus forte valeur ajoutée et vers le trafic par péniches faisant la liaison avec le reste de l'Amérique $\mathrm{du}$ Nord via Seattle (ARRC, 2014). Les résultats financiers de l'ARRC sont fondés essentiellement sur ses activités ferroviaires stricto sensu mais il existe une stratégie de diversification tournée vers la rente foncière - terrains, immobilier, loisirs. La compagnie, dans son plan directeur 2015-2019, l'intègre dans sa stratégie de développement (ARRC, 2015d). L'utilisation de cette rente foncière correspond à la fois à une réponse à la demande en activités touristiques, et à un élément de la stratégie financière de l'entreprise pour diversifier ses revenus (ARRC, 2014; ARRC, 2015c). Le gouvernement fédéral a transféré à l'ARRC des réserves foncières importantes pour permettre la construction de futures infrastructures ou équipements, et pour permettre de générer des revenus fonciers et immobiliers. En plus des $30,5 \mathrm{~m}$ de part et d'autre de la voie, l'ARRC dispose donc de terrains en de nombreux points du territoire, ainsi que des immeubles mis en location. Les réserves foncières de l'ARRC s'élèvent à $146 \mathrm{~km}^{2}$ dont plus de 72 encore disponibles pour des baux à plus ou moins long terme. Les activités immobilières représentent une source de revenus stable pour la compagnie, entre 10 et $13 \%$ selon les années (ARRC, 2015c).

\section{ARRC et tourisme : un acteur de plus en plus engagé}

La compagnie de transport n'est pas uniquement utilisée pour acheminer les voyageurs mais elle devient un acteur du tourisme. Il s'agit là d'une pratique historique aux ÉtatsUnis. En effet, certaines compagnies ferroviaires transcontinentales deviennent des agents touristiques cherchant à valoriser les sites naturels remarquables de l'Ouest américain. L'Atchison, Topeka \& Santa Fe Railroad ouvre le Grand Canyon au tourisme en construisant une desserte ferroviaire et un premier hôtel dans l'Arizona dès les années 1900. Le parc national du Yosemite, créé en 1864, est desservi par un embranchement du Southern Pacific à partir de 1907. La compagnie met en valeur le parc et en fait un produit publicitaire dans le cadre de campagnes promotionnelles (Gordon, 1996 ; Gay, 2006 ; Stilgoe, 2007).

Le secteur touristique est particulièrement dynamique en Alaska. Sur la période octobre 2013-octobre 2014, plus d'1,9 million de touristes extérieurs à l'État ont visité l'État dont 50 \% par le biais de croisières, et les recettes touristiques se sont élevées à 1,83 milliard de dollars. L'essentiel de l'activité touristique se concentre dans le centresud et le sud-est de l'État, là où se situe le réseau ferroviaire alaskien qui participe à l'activité touristique. L'État a retiré 95,6 millions de dollars de revenus de l'industrie touristique dont 24,8 millions issus de l'activité de l'ARRC (Alaska Department of 
Commerce, 2015). Cette stratégie ferroviaire s'inscrit dans un contexte de fort dynamisme du tourisme de montagne et des croisières en Alaska.

L'ARRC s'engage relativement tôt dans une politique touristique qui s'appuie sur ses services de passagers et sur une optimisation de ses possessions immobilières et foncières le long de la ligne : construction d'un nouveau terminal à Denali National Park (1989), construction d'un ensemble hôtelier et d'un parc de caravaning à Fairbanks (1995), reconversion d'un terminal désaffecté à Anchorage en restaurant et en microbrasserie (1996), etc. L'Alaska Railroad Corporation développe cette stratégie dans un but financier pour augmenter ses revenus et dans un but commercial pour attirer davantage de touristes dans ses trains (illustration 3). Elle s'appuie sur l'activité touristique pour modeler ses campagnes de marketing et propose par conséquent une offre touristique intégrée avec plusieurs services réguliers en hiver et en été (illustration 4).

Illustration 3 - La fréquentation de l'ARRC selon ses différents services de passagers de 2010 à 2015 (en nombre de passagers)

\begin{tabular}{|l|l|l|l|l|l|l|}
\hline Service & $\mathbf{2 0 1 0}$ & $\mathbf{2 0 1 1}$ & $\mathbf{2 0 1 2}$ & $\mathbf{2 0 1 3}$ & $\mathbf{2 0 1 4}$ & $\mathbf{2 0 1 5}$ \\
\hline $\begin{array}{l}\text { Denali Star } \\
\text { (service quotidien d'été) }\end{array}$ & 50556 & 56549 & 60041 & 59133 & 60997 & 64304 \\
\hline $\begin{array}{l}\text { Glacier Discovery } \\
\text { (service quotidien d'été et service d'arrêt à la } \\
\text { demande en hiver) }\end{array}$ & 25373 & 27911 & 28921 & 31294 & 34912 & 37987 \\
\hline $\begin{array}{l}\text { Coastal Classic } \\
\text { (service quotidien d'été) }\end{array}$ & 41752 & 43861 & 46015 & 51879 & 53240 & 59417 \\
\hline $\begin{array}{l}\text { Aurora } \\
\text { (service d'été le week-end) }\end{array}$ & 3456 & 3932 & 4841 & 6297 & 7196 & 6500 \\
\hline $\begin{array}{l}\text { Hurricane Turn (hiver) } \\
\text { (service d'arrêt à la demande) }\end{array}$ & 40351 & 46419 & 44960 & 55285 & 54733 & 57241 \\
\hline $\begin{array}{l}\text { Hurricane Turn (été) } \\
\text { (service bihebdomaire) }\end{array}$ & 901 & 1270 & 1416 & 1852 & 1625 & \\
\hline $\begin{array}{l}\text { Grandview Cruise } \\
\text { (service d'été en accompagnant }\end{array}$ & 5695 & 3571 & 3764 & 5809 & 6893 & 10142 \\
\hline $\begin{array}{l}\text { Trains spéciaux } \\
\text { ARRC sous-total }\end{array}$ & 131624 & 146977 & 159160 & 168255 & 175379 & 248545 \\
\hline $\begin{array}{l}\text { Services privés des croisiéristes } \\
\text { des }\end{array}$ & 233160 & 218916 & 211159 & 266105 & 240588 & 226489 \\
\hline
\end{tabular}




\begin{tabular}{|l|l|l|l|l|l|l|}
\hline Fréquentation totale & 405135 & 412312 & 415279 & 489645 & 468661 & 475034 \\
\hline
\end{tabular}

Sources : Alaska Department of Transportation and Public Facilities, 2015 ; Alaska State Rail Plan Draft, op.cit., p. 9-12 ; Alaska Railroad Corporation, 2015 ; Annual Report. Anchorage, ARRC, p. 41-42.

Illustration 4 - L'Alaska Railroad et l'industrie touristique : un axe polarisant ?

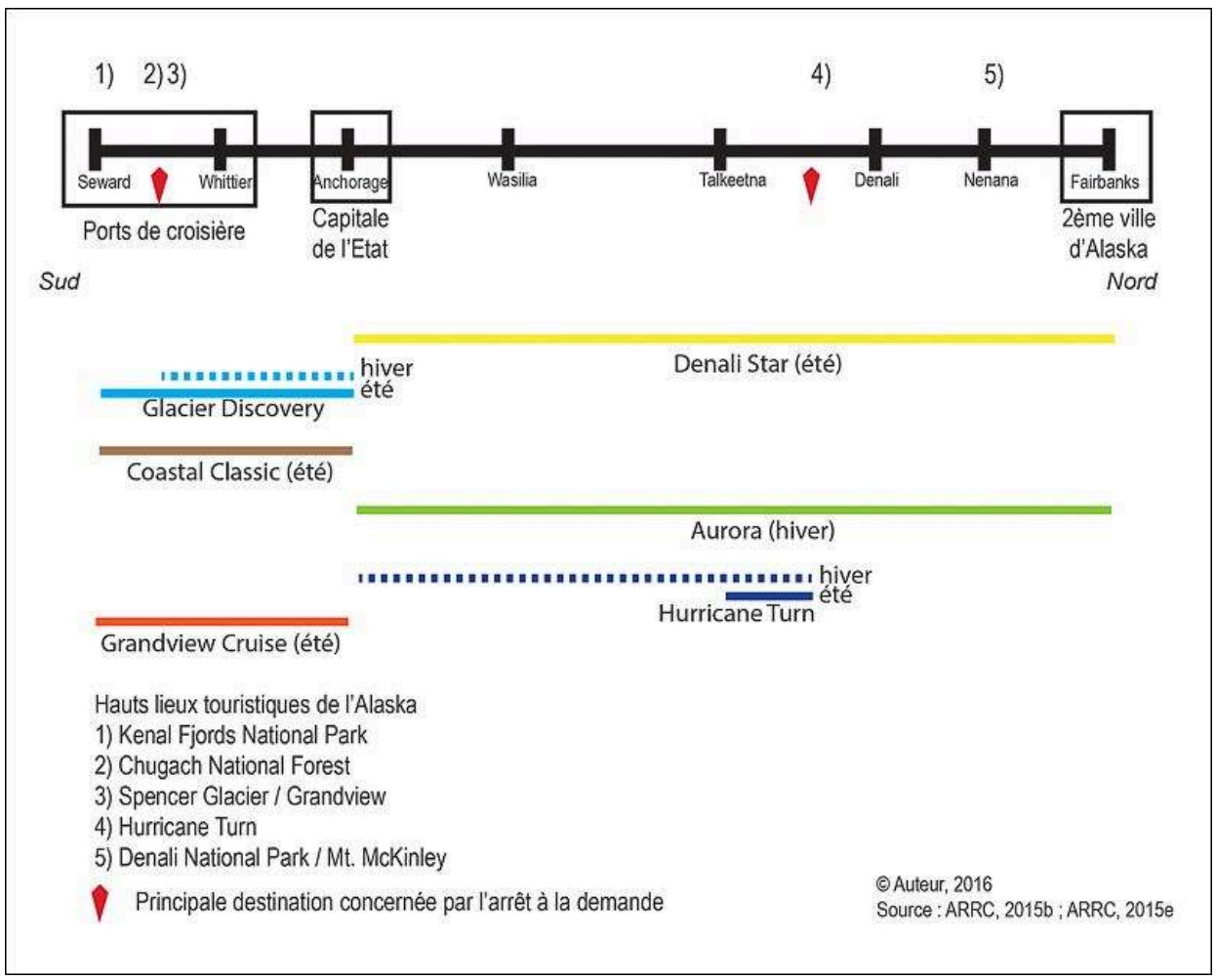

Deux autres éléments viennent compléter cette stratégie touristique. D’abord les services spéciaux pour des événements annuels ou exceptionnels - Easter Train, Great Alaska Beer Train, Alyeska Halloween Train - qui ont pour objectif de capter l'activité touristique ponctuelle. Par ailleurs, l'ARRC assure également des dessertes pour des trains privés des compagnies de croisières. Les accords d'exploitation prévoient que l'ARRC serve d'opérateur ferroviaire aux croisiéristes avec un matériel roulant leur appartenant. Ni le contenu de ces accords, ni ce que cela rapporte à l'ARRC, ne sont précisés dans les rapports annuels et les états financiers transmis à l'État. On peut supposer que ces partenariats avec les croisiéristes, qui représentent plus de la moitié de la fréquentation de la ligne en nombre de voyageurs, assurent un complément de revenus intéressant pour la compagnie. Cette coopération constitue l'un des fondements de la stratégie touristique de la compagnie et l'une des clés pour comprendre sa réussite commerciale et financière.

En 2014, le Denali Star, le Grandview Cruise Train, le Coastal Classic et le Glacier Discovery sont les quatre services les plus fréquentés 9 . Près d'un quart des visiteurs du Denali National Park en 2014 sont arrivés par les trains de l'ARR (ARRC, 2015b). Encore aujourd'hui le transport ferroviaire conserve donc un lien particulier avec les parcs nationaux, ces derniers apparaissant comme des produits d'appel de choix pour une compagnie ferroviaire se développant aussi grâce au tourisme (Gay, 2006). Au-delà de l'importance du tourisme dans le dynamisme ferroviaire alaskien, l'ARRC déploie une 
véritable stratégie touristique intégrée qui propose les billets de train seuls ou des forfaits thématiques, d'une à cinq nuits, comprenant le train, l'hébergement et les activités sur place ${ }^{10}$. Cette compagnie de transport n'est plus seulement un support mais un acteur de la mise en tourisme et de la promotion de l'Alaska.

\section{Conclusion}

L'Alaska Railroad Corporation offre un regard unique sur l'organisation du secteur ferroviaire et sur les perspectives favorables d'une redynamisation du mode ferroviaire pour le transport de passagers. Avec sa gestion unifiée du fret et du transport de voyageurs, son statut d'entreprise propriété de l'État et sa stratégie de diversification de ses revenus et de ses activités, l'ARRC est un modèle à part dans le paysage ferroviaire étatsunien. Le réseau ferroviaire alaskien contribue à l'activité touristique par trois aspects: les déplacements simples des touristes à l'intérieur du territoire, notamment grâce à la desserte des sites remarquables, les partenariats avec les croisiéristes et les offres intégrées proposées par la compagnie elle-même qui comprennent les déplacements et les hébergements. L'ARRC participe d'une ouverture et d'une mise en valeur du territoire de l'Alaska, qui passait avant par les industries extractives, et depuis plus de deux décennies par le tourisme. L'originalité du modèle ferroviaire alaskien tient à la fois à son statut juridique - une compagnie de classe II appartenant à l'État de l'Alaska - et à sa stratégie commerciale. La configuration du réseau, autour d'un seul axe, n'est quant à elle pas différente de ce que l'on observe ailleurs aux États-Unis dans certaines compagnies de fret de classe II et de classe III qui possèdent et opèrent généralement une ligne principale. À partir d'un réseau orienté vers le fret, l'ARRC a réussi à construire et à développer une stratégie intégrant un service dédié aux voyageurs - ce qui n'existe nulle part ailleurs aux États-Unis.

Le regard porté sur cet État aux confins septentrionaux des États-Unis apporte des éléments de réflexion plus généraux: les effets positifs de l'intégration verticale complète avec unité des services, la nécessaire recherche d'une diversification des sources de revenus, la dévolution à l'échelon fédéré d'une compagnie auparavant fédérale ou encore la nécessaire mise en relation du réseau ferroviaire avec le territoire et ses acteurs économiques. L'expérience alaskienne pourrait inspirer certaines compagnies régionales ou locales de fret pour réinvestir dans le transport ferroviaire de passagers là où une demande suffisante existerait. Un parallèle pourrait être dressé entre l'Alaska Railroad Corporation et les services de courte et de moyenne distance d'Amtrak en cours de transfert aux autorités fédérées. Toutefois, la question de l'applicabilité au reste du territoire étatsunien se pose, tant le contexte géographique alaskien est spécifique des points de vue géophysique, économique et urbain. 


\section{BIBLIOGRAPHY}

Alaska Department of a Commerce, Community, and Economic Development, Division of Economic Development, 2015. Economic Impact of Alaska's Visitor Industry 2013-2014. Juneau, ADCCED, $12 \mathrm{p}$.

Alaska Department of Transportation and Public Facilities, 2008. Statewide Long Range Transportation Plan, Let's Get Moving 2030. Juneau, ADOT\&PF, 80 p.

Alaska Department of Transportation and Public Facilities, 2015. Alaska State Rail Plan Draft. Juneau, ADOT\&PF, 154 p.

Alaska Railroad Corporation, 2002. South Central Commuter Rail Network Commuter Study and Operation Study. Anchorage, ARRC, 192 p.

Alaska Railroad Corporation, 2013. Annual Report. Anchorage, ARRC, 47 p.

Alaska Railroad Corporation, 2014. Annual Report. Anchorage, ARRC, 53 p.

Alaska Railroad Corporation, 2015a. Freight Service Business Facts. Anchorage, ARRC, 2 p.

Alaska Railroad Corporation, 2015b. Passenger Service Business Facts. Anchorage, ARRC, 2 p.

Alaska Railroad Corporation, 2015c. Real Estate and Facilities Business Facts. Anchorage, ARRC, 2 p.

Alaska Railroad Corporation, 2015d. Destination Excellence, Strategic Plan 2015-2019. Anchorage, ARRC, $15 \mathrm{p}$.

Alaska Railroad Corporation, 2015e. Winter 2015-2016 Schedules, Fares, Packages. Anchorage, ARRC, $20 \mathrm{p}$.

Alaska Railroad Corporation, 2016. Railroad at a Glance. Just the Facts. Anchorage, ARRC, 2 p.

Association of American Railroads, 2014. A Short History of U.S. Freight Railroads. Washington D.C., AAR, 5 p.

Brehmer E, 2015. Anchorage, Railroad Have Ties that Bind. Alaska Journal of Commerce, juin, p. 2-4.

Clifford H, 1999. Alaska/Yukon Railroads: An Illustrated History. Arlington, OSO Pub., 248 p.

Cohen S, 1984. Rails Across the Tundra. Missoula, Pictorial Histories Publishing Company, 144 p.

Fitch E, 1967. The Alaska Railroad. New York, Praeger, 326 p.

Gale Cengage Learning, 2004. A Brief History of the Alaska Railroad Corporation. In International Directory of Company Histories, vol. 60, Boston, St. James Press.

Gallamore R, Meyer J, 2014. American Railroads: Decline and Renaissance in the Twentieth Century. Cambridge, Harvard University Press, 528 p.

Gay J-C, 2006. Transport et mise en tourisme du Monde. Cahiers de Géographie, Collection EDYTEM, $\mathrm{n}^{\circ} 4, \mathrm{p} .11-22$.

Gordon S, 1996. Passage to Union: How the Railroads Transformed American Life, 1829-1929. Chicago, Ivan R. Dee, 416 p.

Perl A, 2002. New Departures: Rethinking Rail Passenger Policy in the Twenty-First Century. Lexington, The University Press of Kentucky, 352 p.

Madden R, 2005. Alaska. New York, Interlink Books, 317 p. 
Osborne D, 2013. Alaska's Tanana Valley Railroads. Charleston, Arcadia Publishing, 127 p.

Schultz C, 2012. The Span of Alaska's Railways: Modern Transportation, Enduring Piece of History. Alaska Economic Trends, Juneau, Department of Labor and Workforce Development, mars, $6 \mathrm{p}$.

Shaffer M, 2001. See America First. Tourism and National Identity, 1880-1940. Washington \& Londres, Smithsonian Institution, $429 \mathrm{p}$.

Sherwood M, 1967. Alaska and Its History. Seattle, University of Washington Press, 475 p.

Stilgoe J, 2007. Train Time: Railroads and the Imminent Reshaping of the United States Landscape. Charlottesville, University of Virginia Press, $304 \mathrm{p}$.

Thompson L, Kohon J, 2012. Developments in Rail Organization in the Americas, 1990 to Present and Future Directions. Journal of Rail Transport Planning \& Management, vol. 2, n 3, pp. 51-62.

Tuck B, Killorin M, 2004. Economic Significance of Alaska Railroad. Anchorage, Institute of Social and Economic Research, University of Alaska Anchorage, 43 p.

United States Department of Transportation, 2016. Pocket Guide to Transportation. Washington D.C., Bureau of Transportation Statistics, $71 \mathrm{p}$.

Wolmar C, 2012. The Great Railroad Revolution. New York, PublicAffairs, 397 p.

White R, 2014. Alaska Railroad Extension Moves Forward. Alaska Business Monthly, juillet, 3 p.

Site internet de la Federal Railroad Administration (FRA) : https://www.fra.dot.gov/Page/P0548.

Site internet de l'ARRC : https://www.alaskarailroad.com .

Site internet du Business Company History Index : http://www.referenceforbusiness.com/ history2/24/Alaska-Railroad-Corporation.html.

Site internet du Bureau du Recensement fédéral : www.quickfacts.census.gov/qfd/states/ 02000.html.

\section{NOTES}

1. Voir le site internet de la Federal Railroad Administration (FRA) : https://www.fra.dot.gov/ Page/P0548

2. Aux États-Unis, les entreprises ferroviaires sont classées - Class I, Regional (II), Local (III) selon leur taille en termes de volumes transportés, d'étendue du réseau et de niveau annuel de revenus. Cette classification est établie par l'Association of American Railroads : la classe II concerne les revenus opérationnels annuels entre 320 millions et 40 millions de dollars.

3. Voir le site internet de l'ARRC et sa rubrique consacrée à l'histoire de la compagnie : https:// www.alaskarailroad.com/about-us/history

4. White Rindi, 2014. Alaska Railroad Extension Moves Forward. Alaska Business Monthly, $1^{\text {er }}$ juillet.

5. Il existe une petite ligne dans l'extrême sud-est de l'Alaska encore exploitée. Avec près de 395000 passagers transportés en 2013 pendant la saison d'été, il s'agit de l'un des chemins de fer touristiques les plus populaires des États-Unis. Cette ligne, d'une centaine de kilomètres, est aujourd'hui la propriété de la ClubLink Corporation - consortium spécialisé dans le tourisme, l'hôtellerie et les golfs.

6. Voir le site internet de l'Alaska Railroad Corporation, www.alaskarailroad.com/corporate/ AboutARRC/tabid/451/Default.aspx 
7. Population au dernier recensement fédéral de 2010 : www.quickfacts.census.gov/qfd/states/ 02000.html

8. Certaines parties du territoire dans le centre et le centre-sud de l'Alaska ne sont pas accessibles par la route faute d'infrastructures. L'ARRC a donc mis en place un service d'arrêt à la demande - whistle stop/flag stop services. Cela signifie que le train peut s'arrêter, sur demande d'un passager à bord ou se trouvant près de la voie, afin d'embarquer ou de laisser descendre ledit passager. Cette pratique peu courante ne s'applique que pour les services Glacier Discovery et Hurricane Turn.

9. En 2014, l'ARRC dénombre 468661 passagers: Denali Star (60 997), Grandview Cruise Train (54 733), Coastal Service (53 240), Glacier Discovery (34 912), Hurricane Turn (7 762), Aurora (7 196), services spéciaux (10 516).

10. Voir la brochure touristique de l'ARRC concernant les offres disponibles pour la période hivernale 2015-2016. Alaska Railroad Corporation, 2015. Winter 2015-2016 Schedules, Fares, Packages. Anchorage, ARRC, p. 1-20.

\section{ABSTRACTS}

The Alaska Railroad Corporation - ARRC - is a unique railway model in the United States: a public company owned by the State of Alaska, the not-divided freight and passenger services, a multifaceted financial strategy. This rail line has enabled the settlement and the exploitation of Alaska. This transportation mode is nowadays a key actor in the Alaskan transport system. Moreover, its tourism strategy makes the ARRC a leading player in the development of tourism and the enhancement of Alaska. This article aims to analyze the main characteristics of the company and the components of its success and to count the ARRC strategy as a structuring factor of the current economic and territorial dynamics in Alaska.

L'Alaska Railroad Corporation (ARRC) constitue un modèle ferroviaire unique aux États-Unis: compagnie publique détenue par l'État de l'Alaska, unité des services de fret et de passagers, stratégie financière diversifiée. Le chemin de fer a permis l'ouverture au peuplement et à l'exploitation de l'Alaska. Ce mode de transport est aujourd'hui un acteur majeur de son système de transport. Par ailleurs, sa stratégie touristique fait de l'ARRC un acteur à part entière de la mise en tourisme et de la valorisation de l'Alaska. Il s'agit, à travers cet article, d'analyser les caractéristiques propres à cette compagnie et les fondements de sa réussite, et de considérer la stratégie de l'ARRC comme un facteur structurant des dynamiques économiques et territoriales actuelles de l'Alaska.

INDEX

Keywords: Alaska, transportation, railroad, tourism, United States

Mots-clés: Alaska, transport, chemin de fer, tourisme, États-Unis 


\section{AUTHOR}

\section{MATTHIEU SCHORUNG}

Matthieu Schorung, matthieu.schorung@gmail.com est Doctorant en géographie à l'Université Paris-Est - Laboratoire Ville Mobilité Transport (UMR-T 9403). Il a publié récemment :

- Schorung M., 2016. Nouvelle loi transports aux Etats-Unis : monument législatif, intentions de réformes et permanence des déséquilibres fondamentaux. Flux, 2016/1 ( $\left.\mathrm{N}^{\circ} 103-104\right)$, p. 102-106. - Schorung M., sous presse. Le transport ferroviaire interurbain de passagers en Californie : un laboratoire du renouveau ferroviaire aux Etats-Unis ? Flux (accepté, en attente de publication). 